\title{
La investigación formativa en la Facultad de Odontología de la Universidad de Antioquia: vivencias de los estudiantes de pregrado
}

Ángela María Franco-Cortés*, PhD.

${ }_{1}$ Universidad de Antioquia, Facultad de Odontología, Medellín, Colombia

Recibido: 7 de mayo del 2015. Aprobado: 12 de junio del 2015.

*Autor de correspondencia: Ángela María Franco-Cortés. Facultad de Odontología, Universidad de Antioquia, Medellín, Colombia. Calle 64 n 52-59. Teléfono: (57) 421967 72. Correo electrónico: angela.franco@udea.edu.co

Cómo citar este artículo: Franco-Cortés AM. La investigación formativa en la Facultad de Odontología de la Universidad de Antioquia: vivencias de los estudiantes de pregrado. Rev Nac Odontol. 2015; 11(21):37-47. doi: http://dx.doi.org/10.16925/ od.v11i21.946

Resumen. Introducción: el currículo de la Facultad de Odontología de la Universidad de Antioquia está inscrito en una apuesta por la formación de un odontólogo integral con responsabilidad y mentalidad crítica para conocer e intervenir el componente bucal del proceso salud-enfermedad, mediante un modelo pedagógico centrado en procesos participativos y la autonomía del alumno, en el que la investigación es asumida como un eje transversal que debe contribuir a forjar esa actitud crítica y reflexiva ante el conocimiento. El objetivo de este estudio fue reconocer las vivencias narradas por los estudiantes de pregrado de esta Facultad relacionadas con el proceso de investigación formativa, las reflexiones sobre estas vivencias y las lecciones aprendidas. Metodo: desde una perspectiva hermenéutica, se trabajó con una muestra intencional de 53 estudiantes de pregrado matriculados en la última etapa del plan de estudios (octavo, noveno y décimo semestre), quienes se distribuyeron en ocho grupos de discusión. Resultados: de la reflexión que los estudiantes hacen sobre sus vivencias se destacan cuatro temas: la selección y definición del problema a investigar, el acompañamiento de los profesores/ asesores, la relación del plan de estudios con la investigación formativa y el apoyo institucional. Discusión: de modo general, se puede decir que los resultados muestran una serie de desencuentros entre la propuesta curricular y las vivencias de los estudiantes durante el proceso de investigación formativa. Desencuentros que van en contravía de una apuesta pedagógica tan importante como la de un currículo problematizador y flexible que aporte a la formación como proceso y como producto.

Palabras clave: educación superior, estrategias de educación, investigación formativa. 


\title{
Formative Research of the Faculty of Dentistry of the University of Antioquia: Experiences of an Undergraduate
}

\begin{abstract}
Introduction: the Faculty of Dentistry's program is aimed to the formation of a comprehensive dental surgeon with a critical and responsible mindset to know and correct the oral component of the health-sickness process, using a pedagogical model focused on participative processes and the autonomy of the student, in which investigation is understood as the main axe of the formation of that critical and pondering attitude towards knowledge. This study is aimed to recognizing the experiences described by undergraduate students of this Faculty, related to the process of formative research, pondering and learned lessons. Methods: form a hermeneutical point of view, an intentional sample of 53 undergraduate students enrolled in the last stage of the study plan (eighth, ninth and tenth semesters), was used. Such students were distributed in eight discussion groups. Results: out of the students' inputs, four main topics stood out: the selection and definition of the problem to be investigated, the professors/advisors' company, the link between the study plan and the formative investigation and the institutional support. Discussion: in general, the results show a series of inconsistencies among the curricular proposal and the experiences of the students during the formative investigation. These inconsistencies go conversely to the teaching proposal, as important as that of a problematizing and flexible program, which cooperates with the formation as a process and a product.
\end{abstract}

Keywords: higher education, education strategies, formative investigation.

\section{A investigação formativa na Faculdade de Odontologia da Universidade de Antioquia: vivencias dos estudiantes de pré-grau}

Resumo. Introdução: o currículo da Faculdade de Odontologia da Universidade de Antioquia está inserto em uma aposta pela formação de um (a) odontologista integral com responsabilidade e mentalidade crítica para conhecer e intervir o componente bucal do processo saúde - doença, através de um modelo pedagógico centrado em processos participativos e a autonomia do aluno, no qual se assume a investigação como um eixo transversal que deve contribuir a forjar essa atitude crítica e reflexiva ante o conhecimento. O objetivo deste estudo foi reconhecer as vivencias descritas pelos estudantes de pré-grau desta Faculdade, relacionadas com o processo de investigação formativa, as reflexões sobre estas e as lições aprendidas. Métodos: com perspectiva hermenêutica, trabalhou-se com uma amostra intencional de 53 estudantes de pré-grau inscritos na última etapa do plano de estudos (oitavo, nono e décimo semestres), que foram distribuídos em oito grupos de discussão. Resultados: da reflexão que os estudantes fazem em relação a suas vivencias destacam quatro temas: a seleção e definição do problema a ser investigado, o acompanhamento dos professores / assessores, a relação do plano de estudos com a investigação formativa, e o apoio institucional. Discussão: de maneira geral, pode se dizer que os resultados evidenciam uma série de desencontros entre a proposta curricular e as vivencias dos estudantes durante o processo de investigação formativa. Desencontros que se opõem a uma aposta pedagógica tão importante como a de um currículo desafiador e flexível que contribua com a formação como processo e como produto.

Palavras-chave: educação superior, estratégias de educação, investigação formativa. 


\section{Introducción}

El Ministerio de Educación establece que es objetivo de la educación superior y sus instituciones "profundizar en la formación integral de los colombianos, [...] capacitándolos para cumplir las funciones profesionales, investigativas y de servicio social que requiere el país". La Ley 30 de 1992 señala las condiciones que distinguen la universidad de otras instituciones de educación superior, haciendo un llamado a que acrediten "su desempeño con criterio de universalidad en las siguientes actividades: la investigación científica o tecnológica, la formación académica en profesiones o disciplinas y la producción, desarrollo y transmisión del conocimiento y de la cultura universal y nacional" (Artículo 19) [1].

La Universidad de Antioquia, como parte de estas instituciones de educación superior, concibe la investigación como "fuente del saber, generadora y soporte del ejercicio docente", razón por la cual "la investigación junto con la docencia y la extensión constituyen los ejes de su vida académica" [2]. En este contexto, la Facultad de Odontología de la Universidad de Antioquia vela por la formación de un odontólogo integral con responsabilidad y mentalidad crítica para conocer e intervenir el componente bucal del proceso salud-enfermedad, por medio de un modelo pedagógico centrado en procesos participativos y con autonomía del alumno, y en el que la investigación es asumida como un eje transversal que debe contribuir a forjar esa actitud crítica y reflexiva ante el conocimiento [3], [4].

Con esta intención está previsto en el microdiseño curricular del pregrado de esta Facultad que los cursos incluyan actividades de investigación formativa, es decir, actividades que forman parte de la didáctica y cuyo propósito es apoyar la formación integral del estudiante y la adquisición de competencias como las de indagar, criticar, observar, comprender, abstraer, entre otras [5]. Además, el currículo incluye un curso de metodología de la investigación que se va desarrollando desde el IV hasta el $\mathrm{x}$ semestre, $\mathrm{y}$ cuyo producto es un proyecto de investigación que los estudiantes organizados en grupos formulan y ejecutan, y que constituye un requisito de grado.

Esta experiencia - que ya cuenta con más de quince años de desarrollo en la Facultad- ha sido evaluada por los estudiantes de manera normativa, es decir, atendiendo la obligación que tienen los profesores de hacer evaluación de los cursos cada vez que se termina un semestre. Estos procesos evaluativos han hecho cada vez más evidente que la investigación formativa en el pregrado se ha circunscrito a los requisitos del "curso de investigación", y no ha logrado posicionarse como una experiencia significativa y más bien ha entrado en choque, explícita o implícitamente, con otras experiencias de aprendizaje propias del currículo de esta Facultad (preclínicas, clínicas, prácticas académicas, laboratorios, entre otras).

Mediante la pregunta general: ¿cuáles son las vivencias más significativas que caracterizan tu trabajo en el curso de investigación?, este estudio se propuso el objetivo de reconocer las vivencias de los estudiantes de pregrado de la Facultad de Odontología de la Universidad de Antioquia durante el proceso de investigación formativa, además de las reflexiones sobre las dificultades enfrentadas y las lecciones aprendidas.

\section{Métodos}

Este estudio se adelantó desde una perspectiva hermenéutica, dado que interesaba comprender los textos construidos por los estudiantes de pregrado en los que se expresan las experiencias vividas. Gadamer afirma que "la hermenéutica apunta a la comprensión y su tarea no es desarrollar un procedimiento de la comprensión sino iluminar las condiciones bajo las cuales se comprende", con lo cual quiere decir que más que preguntar qué hacer para comprender, con la hermenéutica la pregunta se transforma y lo que importa es cómo es posible la comprensión [6].

En esta investigación, se trabajó con una muestra intencional de estudiantes de pregrado de la Facultad de Odontología de la Universidad de Antioquia, considerando como criterios situacionales para su convocatoria y selección: 1) que estuvieran matriculados en la última etapa del plan de estudios (octavo, noveno y décimo semestre), por ser quienes ya tienen un proceso más avanzado de investigación formativa, y 2) que aceptaran participar voluntariamente en el estudio. La técnica de recolección elegida fue la de grupos de discusión, razón por la cual se conformaron ocho grupos, cada uno de los cuales estaba integrado por 6-8 estudiantes de ambos géneros. 
El acercamiento a los estudiantes y a la manera como ellos cuentan y le dan significado a sus vivencias durante el proceso de desarrollo de su proyecto de investigación se hizo a través de encuentros conversacionales cara a cara, que se realizaron como ejercicios comunicativos en los que se ponían en interacción las reflexiones de los participantes y de la investigadora. Se escogió la conversación porque se le quiso dar preeminencia al lenguaje hablado y gestual, en la medida en que el lenguaje como producto social es útil no solo porque expresa la experiencia subjetiva de los individuos, sino también porque, además, denota el orden social o el contexto en el que se originan los significados.

Sin apuros, se abordaron diferentes temas, según la guía semiestructurada de preguntas elaboradas antes, que recogía las categorías iniciales de interés: la selección del tema de investigación, el acompañamiento del profesor/asesor, la experiencia investigativa en cursos diferentes al de investigación y el apoyo institucional.

La entrevista conversacional se orientó teniendo el mayor cuidado de evitar que las preguntas fueran asumidas como una evaluación sobre el dominio de conceptos; por el contrario, se buscó que generara un clima de espontaneidad y confianza que no inhibiera a los estudiantes para revelar las vivencias y los sentimientos que las acompañaban. Aunque se elaboró una guía de preguntas, se utilizó solo en casos necesarios, pues fue preferible irse adecuando a las respuestas de los participantes dándoles libertad para conducir su discurso y profundizar en él.

Los encuentros se realizaron en espacios cerrados de la Facultad de Odontología; uno o dos con cada grupo y con una duración promedio de 90 minutos por encuentro, con sus ritmos discontinuos y sus circularidades. Todos fueron grabados y posteriormente transcritos. Se solicitó la firma del consentimiento informado que incluía, entre otras, la autorización para grabar la discusión, grabación que solo fue conocida por la investigadora, guardando su confidencialidad.

Como base teórica para la interpretación de las vivencias y de las reflexiones se utilizó el círculo hermenéutico [7], al reconocer que el estudiante -quien tiene unos preconceptos y una estructura cognitiva- se relaciona con la investigación formativa que le propone la Facultad y en esa red de relaciones que establece con el docente, los compañeros y el conocimiento científico, se producen vivencias, sentimientos, pensamientos y se toman acciones frente al proceso investigativo.

El proceso de análisis e interpretación de los discursos producidos por los estudiantes fue permanente y flexible. Inició con el diseño de los grupos, se continuó durante la ejecución y terminó con un análisis descriptivo-narrativo, dado el carácter exploratorio del estudio. A medida que avanzaba la recolección y con base en la lectura, la relectura y la elaboración de resúmenes, se organizaba la información extrayendo de los textos generales aquellos directamente relacionados con el problema de investigación (unidades de significado general), y se clasificaban y codificaban de acuerdo con los temas y las categorías propuestas (unidades de significado relevante).

Después se organizaban las tendencias, con base en la elaboración de memos analíticos, líneas de opinión sobresalientes y contrastación de opiniones entre los grupos y dentro de los grupos, para construir por último los argumentos interpretativos (las vivencias convertidas en experiencias) por parte de la investigadora, de tal manera que se hiciera una traducción del texto construido colectivamente [8].

\section{Resultados}

Cuatro temas se destacan de la reflexión que los estudiantes entrevistados hacen sobre sus vivencias durante su paso por el "curso de investigación" como parte del proceso de investigación formativa. Como ya se dijo, se espera que tenga como resultado, aunque no únicamente, la participación activa del estudiante en la elaboración y ejecución de un proyecto de investigación, bajo la tutoría de uno o dos profesores que juegan el rol de asesores, temático y metodológico.

A continuación, se exponen estos cuatro temas, aunque antes vale destacar que a pesar de que en la conversación se trató todo el tiempo de que las vivencias no se centraran solo en el paso por el "curso" y en el desarrollo del proyecto, los entrevistados volvían permanentemente sobre él. Esto significa que un primer hallazgo del estudio es que para los estudiantes la investigación formativa está asociada solo con esta experiencia; no existe en su imaginario ni en sus vivencias una relación con el contenido o la metodología de los demás cursos del plan de estudios. 


\section{Selección y definición del problema a investigar}

Cuando el estudiante llega al curso de investigación, se hace la inducción y se le asignan las primeras tareas para construir su proyecto; empieza para él un camino, el de seleccionar y delimitar el problema a investigar, el cual emergió como una de las vivencias que más influencia tuvieron en la valoración general de su proceso de formación en y para la investigación. Este camino comienza muy temprano, en el cuarto semestre académico o antes, $y$, por tanto, se ve seriamente afectado por las condiciones particulares que caracterizan al estudiante en ese nivel de su formación como odontólogo: el desconocimiento de la mayoría de los problemas que tiene por resolver la odontología; el escaso manejo de los asuntos disciplinares; poco o ningún contacto con la actividad científica; y en algunos casos, incluso su indecisión acerca de si esta es en definitiva la profesión en la que se quieren formar, entre otras.

De tal manera que la elección, la definición y el planteamiento del problema que debe ser investigado se vive como una etapa abrumadora y de desorientación que representa un comienzo difícil y desconcertante para el estudiante. Este se ve enfrentado a iniciar la actividad científica con poca o ninguna experiencia que trae de su formación secundaria, con la contextualización general que le han hecho en semestres anteriores y en un entorno adverso lleno de obstáculos que van llevando a que las pretensiones curriculares de formar sus competencias investigativas se queden en el papel.

A esta desorientación que el estudiante siente durante la selección del tema y el planteamiento del problema, se suma la poca habilidad y solvencia propia de su nivel académico para argumentar la importancia y la justificación de la elección ante el profesor asesor. Esta vivencia es referida por la mayoría de los estudiantes de manera negativa, en el sentido de que su percepción es que el profesor se ocupa más de descalificar la propuesta que de aportar a su construcción. Por eso, comentan:

G1: “[...] Es que el tema lo elegimos en el tercer semestre, cuando uno no tiene ni idea de odontología, uno no sabe en qué investigar, qué es bueno, qué le puede aportar algo. Fuera de eso cuando a uno se le ocurre un tema, todo se lo tumban [...]"
G4: “¿Preguntas?... uno las tiene todas. Uno no sabe nada de odontología [...]"

G1: "El profesor, que tenía más conocimiento, decía que era una bobada".

G2: “Si, porque uno primero piensa: ¡ay! qué voy a investigar, pues uno cree que todo está investigado, que ya todo se sabe. Y uno piensa ¿qué hago entonces? Es como empezar a buscar a ver qué va a investigar uno y después decirle a los profesores que va hacer eso y ellos te contesten: eso ya está investigado, eso no sirve".

A los estudiantes les impacta tanto esta vivencia, que durante la reflexión varios de ellos propusieron que la Facultad replantee el momento en el que se ven abocados a la selección del tema a investigar o que se replantee la metodología con la que se trabaja este momento de la investigación formativa, de tal manera que se haga menos traumática la experiencia y que esto no vaya menguando su interés por la investigación, como suele suceder.

G2: "No sé cómo hacer, poner la pregunta de investigación en un semestre más avanzado, llegado el caso seguir con la misma metodología, al menos que los estudiantes conozcan algo, ya sea que hayan tenido contacto con los pacientes, si va a ser enfocado como hacia la odontología".

Cabe agregar que a las dificultades en la elección y sustentación del tema se les suma la vivencia, que en muchos casos califican como negativa, de la conformación de los grupos de trabajo (grupos de estudiantes) a partir de la afinidad de los temas escogidos. La Facultad ha probado varias modalidades para agrupar a los estudiantes, incluyendo la de permitir que lo hagan según sus preferencias; sin embargo, también ha recurrido a esta opción de agruparlos por tema elegido, decisión que el estudiante vive como una imposición de trabajar con compañeros que no son los más cercanos, creando desde el comienzo un ambiente de trabajo adverso.

\section{Acompañamiento de los profesores/asesores}

De la manera como curricularmente se tiene dispuesto el acompañamiento que los profesores 
hacen al proceso de investigación formativa, entendido para la mayoría de los estudiantes y profesores como el curso de investigación, se derivan algunas de las más críticas vivencias relatadas por los estudiantes, pues para algunos el trabajo de los profesores que fueron nombrados como sus "asesores" ha sido poco motivador por la actitud pasiva o desinteresada de estos. Los estudiantes captan rápidamente que detrás de esta actitud hay un profesor que cuenta con poca experiencia en investigación, o un profesor al que le fue asignado el "curso" como parte de su "carga" docente pero tiene poca disposición para esta labor. Como es de esperarse, esta actitud del docente se transmite inevitablemente a los estudiantes que están a su cargo y que son muy sensibles a este tipo de conductas, en la medida en que ellos mismos no han sido sensibilizados para entender la importancia de la investigación formativa.

Para otros estudiantes, la vivencia de la asesoría no es positiva, por cuanto los asesores no son seleccionados acertadamente y en algunas ocasiones ocurre que no manejan el tema seleccionado con la adecuada profundidad, lo cual tiene serias consecuencias si tenemos en cuenta que el estudiante tampoco lo conoce. Esta situación se ha tratado de solucionar con la fórmula de nombrar un profesor que se desempeñe como asesor temático y otro que juegue el papel de asesor metodológico, fórmula que en algunos casos funciona y en otros -según lo expresado por los entrevistados- termina creando nuevos problemas, producto de la falta de entendimiento entre estas dos personas.

G7: "[...] muchas veces ellos no saben tampoco del tema escogido, entonces no lo saben guiar a uno".

G5: "Trabajamos solos, nunca tenemos un asesor, solo el profesor de investigación [asesor metodológico] y no más".

Una tercera vivencia relatada por los entrevistados es la del profesor que, a diferencia de los anteriores, asume una actitud de superioridad frente a los estudiantes, con exigencias que en ocasiones no tienen en cuenta su nivel de desarrollo y que termina en imposiciones que los hace sentir "coartados" en su proceso formativo. Los estudiantes plantearon que el asesor temático o el metodológico no tienen en cuenta sus elaboraciones y están cuestionando de manera permanente lo que ellos inicialmente plantearon, lo cual los hace sentir como un actor secundario en el proyecto.

Otra vivencia negativa narrada por los entrevistados es la de los asesores con poco tiempo para atender sus necesidades de acompañamiento y orientación. Igual que en el primer caso, los estudiantes captan la problemática de asesores con un número alto de grupos asignados o de asesores que tienen un plan de trabajo docente muy saturado y que pese a su buena voluntad no logran corresponder con la debida atención y dedicación a los requerimientos -explícitos o implícitos- de los estudiantes. Esto repercute en retrasos del cronograma de trabajo, en una dinámica lenta que no permite hacer una adecuada integración de los momentos del proceso investigativo, y en desmotivación del investigador en ciernes.

Cuestionaron también la falta de "estandarización" de criterios de los asesores para orientar la elaboración del protocolo, lo que se traduce en reelaboraciones interminables del documento, sin que el estudiante capte claramente lo que debe aprender; vivencia que se ve agravada en muchos casos por el cambio frecuente de asesores (hasta tres o cuatro a lo largo de la elaboración y ejecución del proyecto), que ante la falta de un buen "empalme" con el anterior, se suman al trabajo desconociendo lo que se ha avanzado; es decir, se aumenta la dificultad de consolidar una propuesta sobre la que terminan opinando varias personas con criterios disímiles acerca de la viabilidad, la factibilidad y la importancia del tema escogido. Ambas situaciones generan más apatía y alimentan la desmotivación que se ha ido produciendo por la suma de otros obstáculos que han encontrado en este camino, y explican la asociación que en general hace el estudiante de la experiencia de formarse en investigación con múltiples sentimientos negativos:

G1: "[...] y uno encariñarse con la investigación... y de pronto, cambio de asesor, entonces te cambia el tema... entonces uno como que no avanza y le coge pereza y ya no le provoca trabajar".

G8: "Respecto a la redacción del protocolo, como que los asesores no están bien estandarizados, unos quieren documentos muy extensos y muy adornados, y otros los quieren menos complejos, más puntuales... Notábamos que entre los asesores tienen muy diferentes maneras de trabajar este documento". 
Las vivencias narradas permiten entender por qué fue reiterado entre los entrevistados un sentimiento de soledad, a veces expresado con rabia, a veces con impotencia. "Nos sentimos solos" fue una frase que se escuchó muchas veces a lo largo de las entrevistas, y sobre la cual un proceso curricular que ha declarado la investigación como un eje transversal, que teóricamente le ha dado la máxima importancia, debe hacerse muchas preguntas.

Cabe anotar, aunque no fuera frecuente, que también se escucharon voces que reconocieron de manera positiva el acompañamiento del profesor/asesor. Manifiestan que han encontrado en ellos la orientación que esperaban y logran ver la riqueza del proceso de investigación de la mano de su docente, aún sin ver concluidos sus proyectos. En estos alumnos, es evidente la diferencia en la actitud, la comprensión y la motivación que tienen frente a su ejercicio de formación y para la investigación, y no resulta casual que sean ellos quienes mostraron más expectativas sobre la posibilidad de continuar su formación académica con esta proyección.

G6: "Bien... con el asesor metodológico nos ha ido muy bien, nos ha facilitado mucho el trabajo, muy buen guía a la hora de explicar, de hacer el diseño del proyecto, los pasos que hay que seguir en la elaboración. El asesor temático prácticamente complementa el trabajo, nos ayuda mucho a la hora de conceptualizar sobre el tema que estamos estudiando, que en esa parte no ha tenido problemas".

G3: "Pues yo me sentí muy bien... fue súper bueno... él nos sugirió el tema y lo empezamos a desglosar, pero él fue el que nos llevó al tema... él nos dice que nos va a quedar súper bueno, entonces uno va a asesoría y él nos anima muchísimo".

\section{La relación plan de estudios e investigación formativa}

Otra de las vivencias frecuentes en la narración de los entrevistados fue la constante competencia entre el tiempo que demanda el proceso de investigar y la "recarga" de cursos teóricos y clínicos, seminarios y prácticas académicas que caracterizan el currículo de la Facultad, que, entre otras cosas, exige a los estudiantes una excesiva cantidad de horas presenciales que deja poco tiempo para la formación humanista e investigativa. A esto se suma la jerarquización implícita de los cursos que hacen profesores, estudiantes y hasta administradores del currículo, en la que prevalece la formación en clínica odontológica y la subvaloración de aquellos cursos y actividades consideradas "de relleno", entre ellos, la investigación, por cuanto no apuntan directamente a dicha formación clínica. Es decir, la propuesta curricular implica una carga académica que compite no solo en función del tiempo sino también en función de la "importancia" que se le da a cada uno de los cursos para la formación del odontólogo.

G6: "No hay tiempo, aquí las ocupaciones son muchas. Pero sí me gustaría tener un poquito más de tiempo, para poder dedicárselo al trabajo y hacerlo mejor, porque el tema es bueno... es un tema que se puede aplicar luego aquí en la Facultad y que puede crear muchos beneficios a todas las personas".

G2: "Nosotros no queremos graduarnos el año entrante y dejar la investigación en manos de terceros, para mí y para todos sería lo más frustrante que podría pasar".

\section{Apoyo institucional para la investigación formativa}

Otro de los hallazgos importantes son las vivencias que relatan los estudiantes y que resumen como la falta de apoyo de la institución para ejecutar su proyecto, pese a que lo exige como requisito de grado. Mencionan la falta de apoyo económico para financiar los gastos que implica la ejecución, además de la falta de respaldo en las relaciones que se deben entablar con personas o instituciones externas que tienen que ver con la posibilidad de financiar o ejecutar el proyecto. En general, esta es otra situación que alimenta el sentimiento de soledad que caracteriza la mayor parte de las vivencias relatadas.

Los estudiantes se ven enfrentados, pese a que muchos de ellos provienen de familias de bajos recursos económicos, a financiar aquellos costos mínimos necesarios para ejecutar el proyecto y cumplir con los objetivos, aunque como ellos mismos lo relatan, es en ese momento cuando el proyecto se queda parcial o deficientemente desarrollado, generando además un sentimiento de frustración que se suma a los otros ya mencionados. 
G1: "Fracasado, porque al final nos dimos de cuenta que hicimos un trabajo que no sirvió para nada, después de haber cambiado mil veces de tema... entonces eso es algo muy triste".

Las respuestas de los entrevistados permiten comprender que aunque algunos de ellos relatan su experiencia de formación en investigación en sentido positivo, sin duda la mayoría tiene y expresa un sentimiento negativo. De sus comentarios puede interpretarse que, aunque tienen muchas expectativas en los primeros semestres y tienen aspiraciones de hacer un buen trabajo investigativo, todos aquellos conflictos que van apareciendo en el camino producto de una difícil relación con los profesores/ asesores, con compañeros y con el entorno institucional-generan sentimientos negativos con diferentes matices para cada estudiante.

Al revisar las respuestas que los estudiantes dieron a la pregunta ¿qué sentimiento le produce pensar en su experiencia investigativa durante estos años de formación profesional?, la mayoría de las respuestas tuvieron un sentido negativo: cansancio, sufrimiento, inconformidad, preocupación, angustia, desubicación, aburrimiento, estrés, pereza, insatisfacción. Pero también se escucharon expresiones en sentido positivo: motivación, expectativa, curiosidad, satisfacción.

\section{Discusión}

No solola Facultad de Odontología dela Universidad de Antioquia, sino también muchas otras facultades de odontología del país, han adoptado una propuesta curricular que incluye la investigación formativa como un componente transversal del currículo. Esta decisión, al parecer, se ha tomado según la premisa de que es imperativa la presencia de la actividad investigativa atravesando todos los espacios académicos. La discusión a la que exhortan resultados como los anteriores, a directivos y profesores, es la de cómo se ha entendido el asunto de la transversalidad, qué implicaciones pedagógicas tiene esta decisión y qué condiciones académico-administrativas se deben preparar en las instituciones para que se cumplan los objetivos curriculares trazados y la experiencia investigativa de los estudiantes se convierta realmente en una experiencia que potencialice sus facultades para la solución de problemas [9].
De modo general, se puede decir que los resultados de este estudio muestran una serie de desencuentros entre la propuesta curricular y las vivencias de los estudiantes durante el proceso de investigación formativa. Desencuentros que van en contravía de una apuesta pedagógica tan importante como la de un currículo problematizador y flexible que desarrolle la creatividad en alumnos y profesores y que aporte a la formación como proceso y como producto [10]. Por eso, lo más importante es identificar dentro de las instituciones las razones que los explican y emprender planes que mejoren o transformen la situación encontrada.

El primer desencuentro es la decisión de centrar la investigación formativa en un curso de metodología de investigación y la ejecución de un proyecto, y no en la relación educación-investigación. Si bien es cierto que esta es una estrategia que se considera válida, aunque algunos autores se preguntan si se puede hacer investigación en sentido estricto en el pregrado [11], reducirla solo a esta única estrategia es entrar en una serie de contradicciones hasta con la forma misma como esta se define, pues - como lo manifestaron de múltiples maneras los entrevistados- puede generar monotonía y desmotivación, reduce la participación del estudiante en su proceso formativo y no se produce el aprendizaje continuo.

Si la decisión que han tomado las facultades es la de transversalizar la investigación en el currículo, significa que esta se ha asumido como un eje que articula todos los demás componentes curriculares y que su papel es fundamentalmente pedagógico, es decir, es la pieza clave de un currículo en el que las dinámicas investigativas se convierten en medios que activan la enseñanza y el aprendizaje [12]; un currículo problematizador, como lo llaman algunos autores, a través del cual se desarrollen competencias comunicativas e investigativas para un ejercicio profesional reflexivo y comprometido con la transformación de la realidad [9].

Salirse de la zona de confort no es fácil para las directivas universitarias, los profesores y los estudiantes. La transversalización de la investigación requiere contextos pedagógicos que faciliten una relación activa y constructiva con el conocimiento; requiere de decisiones político-administrativas que por lo general son traumáticas y demandan recursos. Hacer docencia investigativa requiere un esfuerzo pedagógico y didáctico que, en muchas ocasiones, los profesores no están dispuestos a 
asumir o, cuando lo están, no reciben apoyo de los administradores; y por el lado de los estudiantes, cuando ellos no están familiarizados con las pedagogías activas, y esto en nuestro medio ocurre con frecuencia, su mente se resiste inicialmente a ellas y solo con un trabajo persistente encuentran sus bondades.

Un currículo que le apueste a un modelo pedagógico que desee salirse de lo tradicional y del conductismo debe centrase en el sentido de la experiencia, reconociendo que el conocimiento se construye de múltiples maneras, a través de las experiencias vividas en el aula, en todos los cursos y no solo en el de "investigación". En otras palabras, debe ser un currículo que se fundamente en el aprendizaje significativo, en el que el sujeto aprende a través de un proceso individual y colectivo de construcción de nuevos conocimientos sobre los que ya poseía, y que se van modificando; un proceso en el que la estructura mental se va transformando.

Lo anterior supone un compromiso de todos los estamentos que tienen que ver con la cultura académica. Que el propósito de formarse y formar en y para la investigación se desarrolle de acuerdo con lo que propone la calidad de la educación en Colombia, en la que se hace un énfasis especial en el desarrollo de procesos curriculares con enfoque investigativo [13]. Un compromiso que, por tanto, no es solo del profesor, que necesita voluntad política, respaldo y condiciones académicas y administrativas institucionales para aplicar una docencia más creativa; pero que sin embargo, y de acuerdo con lo expresado por los entrevistados, sí tiene en él uno de sus soportes más importantes.

A pesar de lo anterior, las vivencias relatadas por los estudiantes son contundentes en señalar que para ellos, en la mayoría de los casos, la participación de los profesores, sea tutor o no de su proyecto, es pobre, genera poca motivación y contradice los objetivos de la investigación formativa, pues les coarta la iniciativa y la creatividad. Es decir, que para los estudiantes, como orientadores y responsables del proceso pedagógico y de la formación, el docente es un gran ausente, no físicamente sino pedagógicamente.

Varios autores reflexionan sobre este tema [14], [15], [16] y consideran importante señalar que, en muchos casos, aunque los profesores tengan la voluntad de hacer de la docencia algo diferente, asuntos de variada naturaleza entran en choque con la realidad e impiden avances reales en la orientación del proceso educativo: por un lado, el predominio, aún en los claustros universitarios, de un modelo formativo del que los profesores, alguna vez, también formaron parte como alumnos, basado en una pedagogía netamente transmisionista; por otro lado, un currículo poco flexible y una institución que no ha reflexionado sobre las necesidades en recursos humanos, físicos y materiales para poder hacer realidad la decisión tomada.

Sobre lo primero habría que decir que la docencia transmisionista, que hace al maestro protagonista de la enseñanza y reproductor de saberes, compite fuertemente con las pedagogías activas que exigen del profesor más creatividad, una relación más horizontal con el alumno, primacía del aprendizaje sobre la enseñanza, flexibilidad con los resultados del proceso educativo, didácticas diversas y evaluación abierta [17]. Es decir, exigen del profesor una formación que en muchos casos no tienen aquellos que se desempeñan como tal con solo su formación profesional.

En cuanto a los recursos humanos, físicos y materiales, termina siendo una paradoja que en un contexto como el de las universidades públicas, cada vez más deficitarias financieramente, con menos profesores vinculados y más de cátedra, y con una infraestructura que se va quedando corta y obsoleta, sea donde se esté haciendo con mayor intensidad el llamado a la adopción de pedagogías activas y de didácticas que no se limiten, en el caso de las competencias investigativas, a un curso de metodología del que se hacen cargo dos o tres profesores mientras los demás se encargan de reproducir las didácticas transmisionistas.

Las instituciones deben apoyar a sus docentes para que la práctica pedagógica en general se asuma como investigación, o lo que se conoce como docencia investigativa. De tal manera, según la orientación del docente de cada curso, que plantea situaciones problemáticas, estimula el proceso cognitivo y activa procesos de pensamiento, el estudiante toma la iniciativa de buscar el conocimiento (aprendizaje por descubrimiento). No basta, como en muchos casos lo denunciaron los entrevistados, que el profesor asuma el papel de "revisor" de los avances del proyecto que formulan los estudiantes; es necesario que todos los profesores que participan en los diferentes cursos implementen estrategias didácticas que los "seduzcan" y promuevan en ellos el desarrollo de competencias en el aula; estrategias como el aprendizaje basado en problemas, el 
seminario, la elaboración de estados del arte, entre otros; en general, estrategias didácticas que potencien las habilidades para un pensamiento crítico, reflexivo y creativo.

Dicho todo lo anterior, no cabe duda de que la investigación formativa mediante la docencia investigativa no es una decisión que solo requiere quedar establecida en el papel. Las instituciones, en este caso las facultades de odontología, tienen que saber que no basta con que esta intención quede escrita de manera "impecable" en sus apuestas curriculares, sino que también son necesarias condiciones de la administración curricular, del plan de estudios, del recurso humano (profesores e investigadores y sus comunidades académicas), de infraestructura, de recursos financieros, que den soporte a esta intención y que garanticen un adecuado seguimiento y evaluación de su desarrollo, de tal manera que los correctivos sean aplicados en el momento en que se necesiten. Con la garantía de estas condiciones, vivencias negativas como las narradas por los estudiantes sobre "la ausencia del profesor" y la "falta de apoyo institucional" pueden ir haciendo tránsito hacia vivencias positivas.

\section{Conclusión}

En el desarrollo de la investigación formativa, existen dificultades evidentes. En el caso específico estudiado, podría decirse que ha imperado el estereotipo de la investigación como curso, con un excesivo acento en el objetivo de que los estudiantes aprendan el método científico y con una inducción al mundo de la ciencia en la que ha prevalecido más la mirada instrumental que una reflexión inicial sobre su papel en el desarrollo humano, y una mirada crítica sobre el compromiso ético y político del profesional y del investigador.

Con algunas escasas excepciones de estudiantes que valoraban la experiencia, que se sentían motivados y reconocían la importancia de la investigación formativa para su desarrollo personal y profesional, la gran mayoría narró vivencias muy adversas que se correspondían con acentuados sentimientos de desconcierto, apatía y desmotivación. Esto los inducía a rechazar la idea de la investigación como competencia necesaria para participar activamente y con liderazgo en la búsqueda de soluciones a las problemáticas de su vida profesional y social, y la idea de transformación de la realidad colombiana en salud y en salud bucal caracterizada por la inequidad y la injusticia.

El modelo pedagógico de procesos conscientes, del cual se habla en el marco conceptual de la Facultad de Odontología de la Universidad de Antioquia, se enmarca dentro de la propuesta de educación desarrollista, en la que los estudiantes no son ajenos a su proceso de aprendizaje, de educación y de formación, propuesta que se centra en la problematización y que tiene como una de sus estrategias didácticas la docencia investigativa. Por tanto, el ejercicio de una verdadera investigación formativa debe ser una preocupación y un objetivo constante de los propósitos del proyecto educativo para que conduzca ciertamente a su formación integral como ciudadanos, personas y profesionales [18].

\section{Referencias}

[1] Colombia, Congreso de la República. Ley 30, por la cual se organiza el servicio público de la Educación Superior (28 dic 1992).

[2] Universidad de Antioquia. Acuerdo superior 2004 del 6 de noviembre del 2001.

[3] Mejía R, Jaramillo G. La investigación en el pregrado. Compilación. Documento aprobado por el Consejo de la Facultad de Odontología de la Universidad de Antioquia N. ${ }^{\circ} 124$ del 11 de noviembre de 1998.

[4] Universidad de Antioquia. Facultad de Odontología. Marco Referencial. Programa de Pregrado en Odontología. Febrero 2001.

[5] Ossa J. Formación investigativa versus investigación formativa. Uni-Pluri-Versidad. 2002;2(3):49-56

[6] Gadamer HG. Verdad y método I. Fundamentos de una hermenéutica filosófica. Salamanca: Sígueme; 1993.

[7] Taylor SJ y Borgan R. Introducción a los métodos cualitativos de investigación. La búsqueda de significados. Barcelona: Paidós; 1992.

[8] Galeano ME. Estrategias de investigación social cualitativa. El giro en la mirada. Medellín: La Carreta; 2004.

[9] González E. Un recorrido por los modelos pedagógicos a través de sus didácticas. Análisis de Problemas Universitarios. 1999; 25:58-68

[10] Galeano J. La práctica curricular: entre un interés técnico-práctico y emancipatorio. Cuadernos Pedagógicos. 2006;23:57-88

[11] Restrepo B. Conceptos y aplicaciones de la Investigación Formativa, y criterios para evaluar la inves- 
tigación científica en sentido estricto. Disponible en: http://www.cna.gov.co/1741/articles-186502 doc_academico5.pdf

[12] Jaramillo J. De la investigación formativa a la formación en investigación. Lectiva, 2004;6-7:173-9.

[13] López N. Currículo y calidad de la educación en Colombia. Bogotá: ICFEs; 1989.

[14] Hernández CA. Investigación e investigación formativa. Nómadas, 2003;18:183-93.

[15] Rojas M. Investigar la investigación. La práctica docente en la enseñanza de la metodología de la investigación en la Universidad de Ibagué: Ibagué: Universidad de Ibagué; 2005.
[16] Rojas M. La investigación formativa, sustento y tensión de la docencia en la universidad. Notas universitarias, 2006;16:35-45.

[17] Castañeda B. y Ossa J. (Ed.). Por los caminos de los semilleros de investigación. Medellín: Biogénesis, Fondo Editorial Universidad de Antioquia; 2005.

[18] Tovar M. El significado de la formación integral en estudiantes de último semestre de la Facultad de Salud de la Universidad del Valle, 2002. Colomb Med. 2002;33:149-55. 